\title{
Nano-imprinted subwavelength gratings as polarizing beamsplitters
}

\author{
Julian Wüster ${ }^{\text {* }}$ (D), Yannick Bourgin ${ }^{1,2}$, Patrick Feßer ${ }^{1}$, Arne Behrens ${ }^{1}$ and Stefan Sinzinger ${ }^{1}$
}

\begin{abstract}
Polarizing beamsplitters have numerous applications in optical systems, such as systems for freeform surface metrology. They are classically manufactured from birefringent materials or with stacks of dielectric coatings. We present a binary subwavelength-structured form-birefringent diffraction grating, which acts as a polarizing beamsplitter for a wide range of incidence angles $-30^{\circ} \ldots+30^{\circ}$. We refine the general design method for such hybrid gratings. We furthermore demonstrate the manufacturing steps with Soft-UV-Nanoimprint-Lithography, as well as the experimental verification, that the structure reliably acts as a polarizing beamsplitter. The experimental results show a contrast in efficiency for TE- and TM-polarization of up to 1:18 in the first order, and 34:1 in the zeroth order. The grating potentially enables us to realize integrated compact optical measurement systems, such as common-path interferometers.
\end{abstract}

Keywords: Subwavelength-structures, Polarizing diffraction grating, Nanoimprint-Lithography

\section{Introduction}

Precision optical measurement systems like interferometers consist of a light source, a detector, and the optics, usually including a beamsplitter. It separates the path of illumination and detection from each other and helps to avoid an oblique setup or shadowing effects. The classical configuration with a polarizing beamsplitter leads to two different optical axes, which makes the overall system less compact and difficult to align. In order to avoid these challenges, gratings have been suggested as beamsplitters in measurement systems. An example of a system for measuring freeform surfaces which has been suggested by Bichra et al. $[1,2]$ is shown in Fig. 1.

In this case, light from the source is collimated and passes the grating. The $0^{\text {th }}$ diffraction order is used for illumination, the higher orders are filtered. As the reflected light passes the grating again, just the higher orders carrying the information about the surface are used

\footnotetext{
*Correspondence: julian.wuester@tu-ilmenau.de

'Department Technical Optics, Institute for Micro- and Nanotechnologies,

Technische Universität IImenau, 98693 IImenau, Germany

Full list of author information is available at the end of the article
}

for the detection on the sensor. This allows one to maintain a single optical axis over the whole system. However, a significant fraction of the light intensity is lost due to the two filtering steps. A solution to this is the use of a grating with specific polarization-dependent diffraction efficiencies. The schematic of this concept is illustrated in Fig. 2.

Throughout this paper the diffraction efficiency $\eta$ is defined as the intensity of the respective diffraction order relative to the intensity of the incoming light. The transversal electric (TE) component of the light wave vector is oriented parallelly to our grating structure and the transversal magnetic component (TM) perpendicularly to our structure. For TE-polarization the $0^{\text {th }}$ order carries all the transmitted intensity, so $\eta_{T E, 0} \rightarrow 1$. The light interacts with the specimen between two passes of the quarter-wave plate, resulting in a rotation of the polarization plane by $90^{\circ}$. For this TM polarization in the return path, the diffraction efficiency is $\eta_{T M, 0} \rightarrow 0$. Thus, the light is diffracted solely into higher orders. This effectively makes the grating a compact polarizing beamsplitter, possibly consisting of only one material. As we continue 


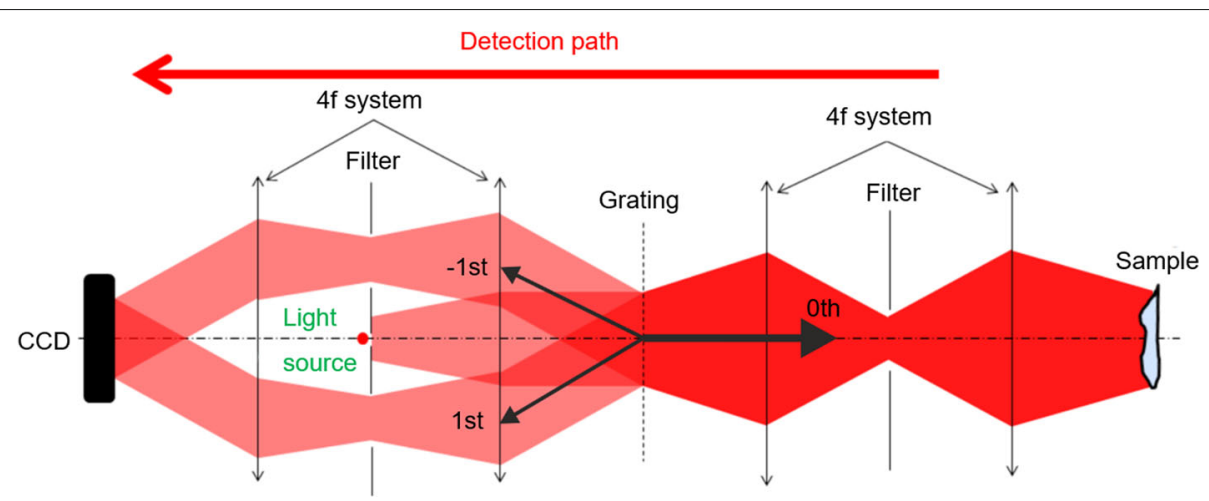

Fig. 1 Uni-axial measurement setup for freeform surfaces. [2]

with the design of such a grating, we keep the following requirements in mind:

1 Diffraction efficiency $\eta_{T E, 0}>>\eta_{T M, 0}$

$\left(\eta_{T M, 1}>>\eta_{T E, 1}\right)$

2 Functionality over a large range of incidence angles $\theta_{i}$

3 Manufacturability and reproducibility (cost-effectiveness)

\section{Tolerance to manufacturing errors}

The tolerance to a large range of incidence angles is necessary in order to enable the measurement of surface profiles, which reflect the light not solely perpendicularly.

Various approaches have been suggested for the design of diffractive structures, which act as polarizing beamsplitters. In the following we will first give an overview over the existing structures which have been used, both with subwavelength features and larger structures. Then we will get to the design process of our hybrid subwavelength-structured diffraction grating, which includes both the effective medium approximation
(EMA) and rigorous coupled-wave analysis (RCWA) [3]. The general method can be applied to other diffractive elements with smaller structures. Afterwards we will present our in-house manufacturing process including the Soft-UV-Nanoimprint-Lithography [4] before we discuss the experimental verification.

\section{State of the art}

There are different approaches for designing a polarizing beamsplitter. Classical methods use naturally birefringent material or dielectric coatings. Here, we focus on a different approach: The use of gratings for achieving a polarizing beamsplitter functionality. There is a large variety of how to realize subwavelength or resonant structure based beamsplitters. Following this definition, resonant structures are of the same magnitude as the wavelength, but allow more than just the $0^{\text {th }}$ order to propagate (although the functionality of subwavelength structures can of course be explained through resonance effects). In this paper we just consider dielectric media, as we want

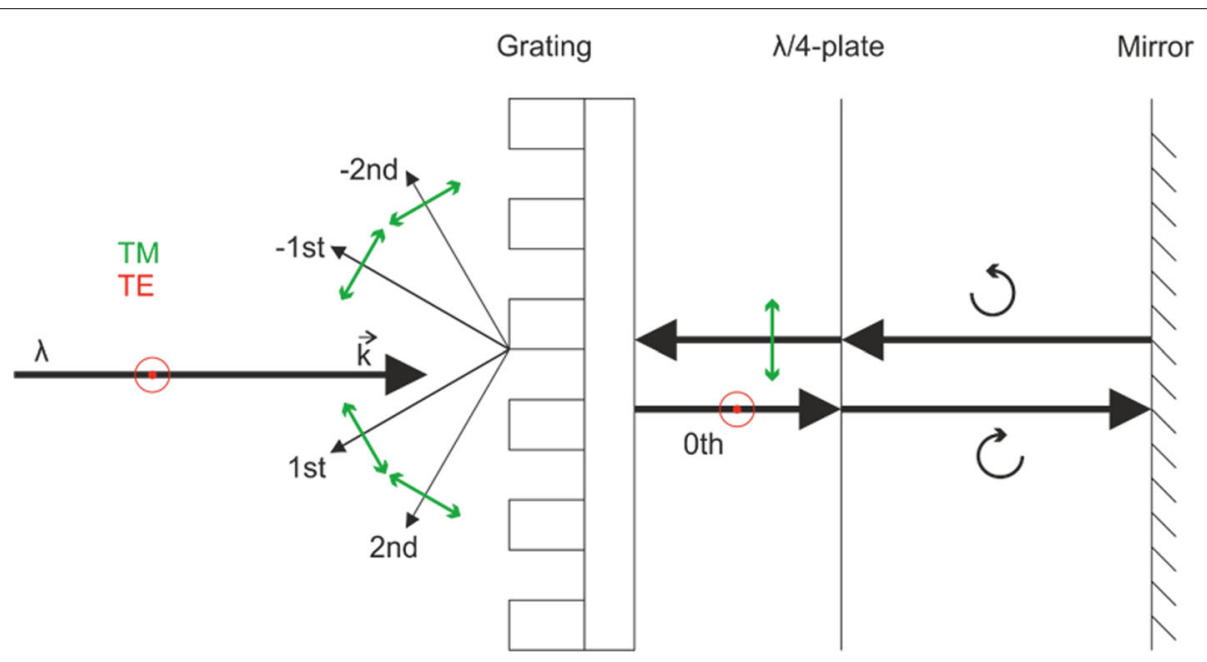

Fig. 2 General concept for the grating 
to design an efficient transmission grating. Metallic gratings (such as e.g. wire grid polarizers) have a lower overall transmission efficiency than dielectric phase gratings.

Several examples have been suggested for grating beamsplitters working under Bragg (or Littrow) incidence angles. The grating period $p$ in this case is $\approx \lambda$. Under this specific angle it is possible to choose a parameter set (period, wavelength, fill factor, which is the ratio between the structure width and the period) in such a way, that either the $0^{\text {th }}$ or $1^{\text {st }}$ order will be transmitted for the different states of polarization. This has been shown for instance by Bartelt et al. [5] and has been theoretically investigated further e.g. by Clausnitzer et al. [6]. This is also possible for the $2^{\text {nd }}$ or higher diffraction order [7].

Subwavelength structures can be described as an effective medium, whose refractive index depends on the geometrical parameters of the designed element. The potential use as anti-reflective surfaces or their use for wave-plates due to their form-birefringence were first described by Enger et al. [8]. Demonstrating the effects on a large scale with water waves, a blazed diffraction grating solely consisting of binary structures was designed by Stork et al. [9]. The possibilities using the effect of the form-birefringence of those subwavelength-structures were further investigated and described by Haidner et al. [10] and Lalanne et al. [11]. Advances in manufacturing (electron beam lithography) led to the demonstration of a blazed grating for the visible spectrum $[12,13]$. Those binary effective media blazed gratings possibly relief the stress of fabrication in comparison to conventional blazed gratings, for which a slope has to be realized.

Subwavelength structures can show polarizationdependent refractive indices. This is the so-called form-birefringence, which, depending on the material and design parameters, can be much higher than natural birefringence. This can be used to realize quarter-wave plates, as described by Cescato et al. [14], which can also be achromatized to some extent [15]. This was demonstrated for the infrared regime [16], and with advancement of different manufacturing techniques also for visible light with different materials [17]. Due to the necessary high aspect ratio the manufacturing is a challenge and is carried out with electron beam lithography. However, there are approaches of reducing the cost of reproducing those form-birefringent quarter-wave plates [18]. Using the same principle, also half-wave plates have been realized [19] (in titanium dioxide, $\mathrm{TiO}_{2}$ ). This can be generalized in principle to any arbitrary relative phase delay.

There are also structures of mixed functionality. Those are more complex structures, which consist of several layers of different materials. Lopez et al. [20] show a device, which acts as a quarter-wave plate under normal inci- dence and as a polarizing beamsplitter under an incidence of $\approx 40^{\circ}$, which is similar to the resonant Bragg-mounted gratings discussed before.

There are also solutions for designing a beamsplitter, which use different stacked materials. For instance, Tyan et al. [21] demonstrate a grating, which transmits TMpolarized light and reflects, even though it is not metallic, TE-polarized light under an incidence angle of $\approx 42^{\circ}$. For just two layers $\left(\mathrm{TiO}_{2}\right.$ on glass) a beamsplitter was demonstrated, which transmits both TE and TM-polarized light. It works for an angle of incidence of $45^{\circ}$, transmits the TE-polarized light without deviation, but the transmitted TM-polarized light is deflected by $90^{\circ}$ [22].

If we also consider circularly polarized light, a number of possibilities to design gratings acting as beamsplitters or -switches have been proposed and demonstrated by Hasman et al. [23].

For our needs, we would like to work with linearly polarized light and need both TE- and TM-polarized light to be transmitted. Several methods for designing gratings acting as polarizing beamsplitters under normal incidence in a way that either the $0^{\text {th }}$ or the higher orders are efficient, have been discussed and calculated both with EMA and RCWA in [24]. There is also an example for such a grating manufactured with electron beam lithography in gallium arsenide, which shows very high differences in diffraction efficiency [25].

\section{Design concept}

The aim is to design a grating, which has ideally an overall efficiency $\eta_{T M, 0}=0$ and $\eta_{T E, 0}=1$. It is well-known, that binary phase gratings with a phase-depth of $\phi=\pi$ (or integral even multiples) diffract all the light into the higher orders and gratings with a phase-depth $\phi=2 \pi$ (or integral multiples) do not diffract the light, so all the light remains in the $0^{\text {th }}$ order. Therefore, realizing a phase difference of $\psi=\phi_{T E}-\phi_{T M}=\pi$, we combine a conventional super-wavelength diffraction grating with polarization-dependent subwavelength structures. To this end we follow and refine the design strategy suggested in [24]. We aim for a design and qualitative phase differences illustrated in Fig. 3.

For the polarization-dependency one needs to choose the material and geometrical parameters of the subwavelength structures. This can be done with the effective medium approximation (EMA). While not requiring large computational effort, it already provides a lot of information on the grating structure and design restrictions. This process is described in the following section. For calculating the efficiencies, one can apply rigorous calculations such as RCWA (rigorous coupled wave analysis). It also can be used to check the accuracy of the EMA calculations and calculate the effect of deviations. 

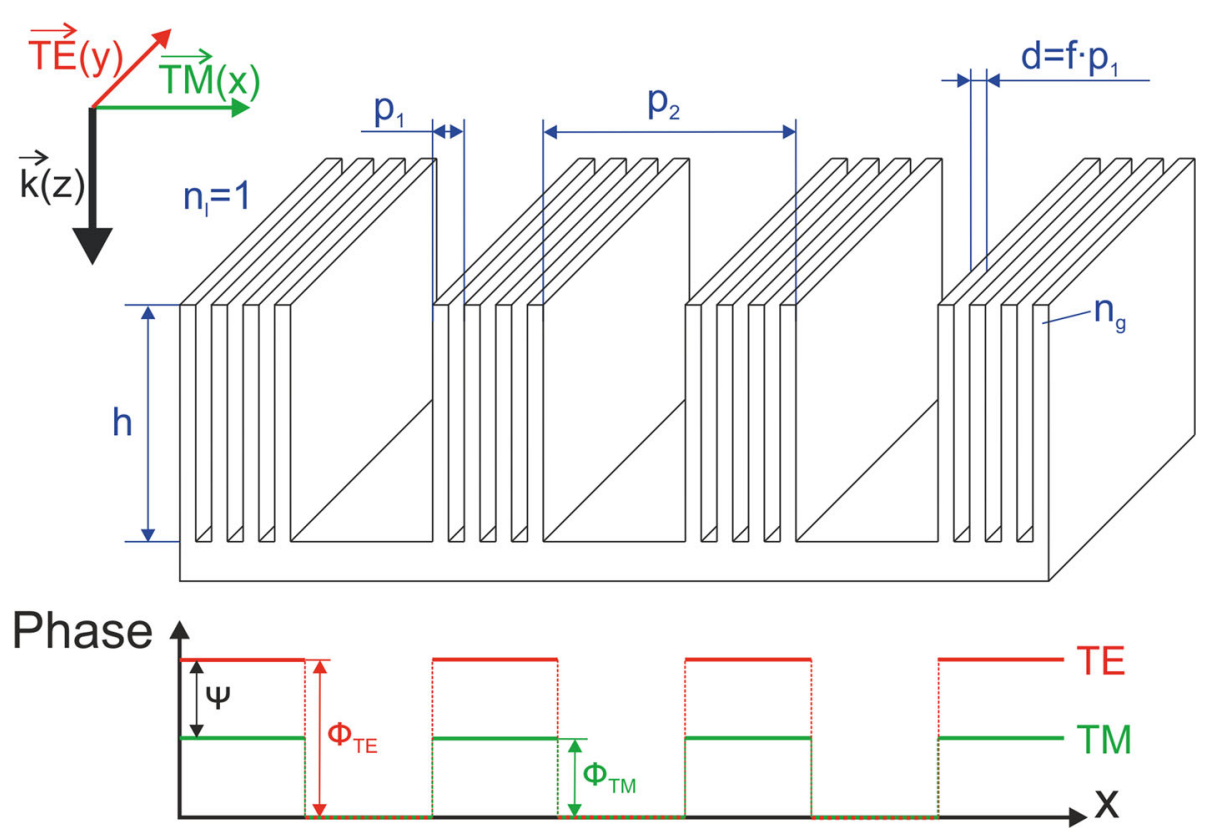

Fig. 3 Final design

\section{Choice of material and fill factor}

As we shrink down the period of a binary one-dimensional grating, it no longer acts as a classical diffraction grating but as a zero-order grating. This means, that there are no propagating diffracted orders, all orders but the $0^{\text {th }}$ are evanescent. This is the case for periods $\mathrm{p}$ :

$$
p \leq \frac{\lambda}{n_{s}+n_{l} \sin (\theta)}
$$

where $\theta$ is in the angle of incidence, $n_{s}$ the refractive index of the substrate, $n_{l}$ the refractive index of the surrounding medium and $\lambda$ the wavelength. The parameter $f$ is the fill factor (or duty cycle), the ratio of the width of the ridge $d$ and the period $p$. Depending on the fill factor of such a grating, it can potentially introduce so-called form birefringence, i.e. it has different refractive indices for different states of polarization. If we shrink down the period to an extension of $p<<\lambda$, we can apply the EMA to model the different refractive indices and calculate the expected phase shifts in the $0^{\text {th }}$ order.

We use the simple first-order EMA-approach given in Eq. 2.

$$
n_{T E}=\sqrt{f \cdot n_{g}^{2}+(1-f) \cdot n_{l}^{2}} \quad n_{T M}=\frac{1}{\sqrt{\frac{f}{n_{g}^{2}}+\frac{1-f}{n_{l}^{2}}}}
$$

Note, that according to [26, 27] this only leads to good results with deviations in reflectivity of the effective medium $<1 \%$ for small periods $(p<\lambda / 40)$. Haidner [27] shows, that Eq. 2 in comparison to RCWA yields too small values for the refractive index for TM. He also offers correctional terms for larger periods. Without this correction, the deviation increases as the period becomes larger. However, for an initial estimation for the first set of parameters, Eq. 2 have proven useful during our approach up to roughly values of $p=\lambda / 4 \ldots \lambda / 3$.

For a grating operating in air as surrounding medium, there are two design parameters, the fill factor $f$ and the grating material with the refractive index $n_{g}$. Figure 4 shows the form birefringence $\Delta n=n_{T E}-n_{T M}$ for two standard materials in lithography, silicon and fused silica. Additionally, the relationship is shown for titanium dioxide, which is a high-refractive material, that is highly transparent for the visible spectrum.

The curves in Fig. 4 clearly show, that the form birefringence heavily depends on the refractive index of the material, which makes choosing the right (highly refractive) material crucial for the design process. Bearing in mind the manufacturing process, a high $\Delta n$ is preferable. The higher $\Delta n$, the less deep the structures eventually need to be etched in order to achieve the desired phase difference of $\pi$ for the orthogonal polarization directions. The parameter to describe this is the aspect ratio $A R$, which is the ratio of the characteristic structure size and the etching depth (structure height), in our case $A R=d / h$. With regard to the technological realization of subwavelength gratings, the aspect ratio $A R$ has to be limited. The etching of both extremely small and at the same time deep structures poses extraordinary challenges on the fabrication process. Therefore, one needs to choose a material and a fill factor $f$ in a combination that allows for relatively small aspect ratios $A R$. There are two general approaches to 


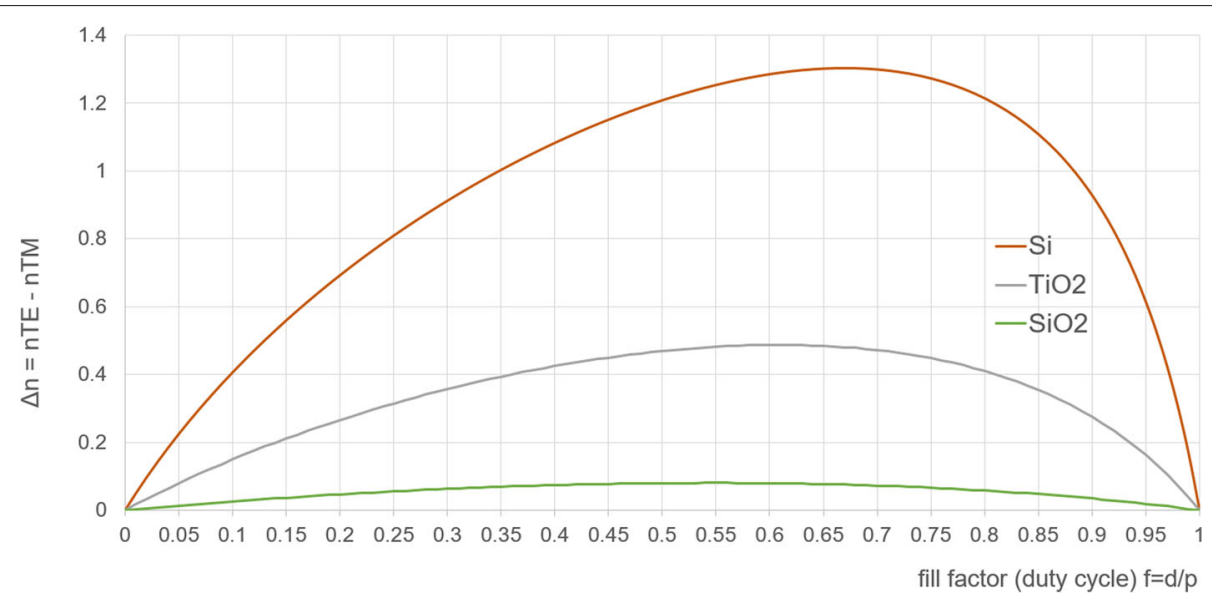

Fig. 4 EMA calculations $\Delta n(f)$

this challenge: We either yield the lowest aspect ratio for $f=0.5$, because for other fill factors the width $d$ of the ridges or the trenches, respectively, becomes smaller. Alternatively, as we can see in Fig. 4, we reach a maximal $\Delta n$ for $f_{\max }=0.67$ for silicon, $f_{\max }=0.61$ for titanium dioxide, and $f_{\max }=0.55$ for fused silica. To directly compare the potential sets of parameters in terms of manufacturability, one needs to calculate the aspect ratio.

To this end, we need to choose the wavelength $\lambda$, the period $p$, and calculate the etching depth $h$, which leads to a phase shift $\psi=\phi_{T E}-\phi_{T M}=\pi$.

$$
\begin{aligned}
\psi & =\phi_{T E}-\phi_{T M}=\frac{2 \pi}{\lambda} \cdot h\left(n_{T E}-1\right)-\frac{2 \pi}{\lambda} \cdot h\left(n_{T M}-1\right) \\
& =\frac{2 \pi}{\lambda} \cdot h \cdot \Delta n
\end{aligned}
$$

Generally speaking, the larger the wavelength, the larger the period for a zero-order grating and the easier the manufacturing. But there is a trade-off, because a larger wavelength leads to the need for deeper structures (see Eq. 3). Different wavelengths both in the visible and near IR regime were considered. The two wavelengths $\lambda=$ $700 \mathrm{~nm}$ and $\lambda=1400 \mathrm{~nm}$ in Table 1 are shown exemplarily for those regions. The $1400 \mathrm{~nm}$ were chosen with regard to our tunable laser source in our experimental setup. The parameter study in Table 1 shows EMAcalculations for the three materials shown above at a maximal period at which our straightforward EMA-approach gives still meaningful results and a significant form birefringence can be expected. The aspect ratio was calculated $A R=\frac{h}{p(1-f)}$, as the smaller lateral structure (ridge or trench) determines the manufacturing limits. Note, that silicon is not transparent for visible wavelengths.

Assuming that we could achieve $A R$ aspect ratios of up to 3-5 in our manufacturing approach it becomes clear, that fused silica is not a suitable material for our demands. The required $A R$ is simply too large for such small periodic structures. This is unfortunately the case for most glass materials, as they usually have relatively low-refractive indices. We also see that even though choosing $f_{\max }$ lowers the required etching depth, it increases the technological challenges concerning the aspect ratio. For silicon (with an assumed maximum aspect ratio of $A R=4$ and a wavelength of $\lambda=1400 \mathrm{~nm}$ ) it would be possible to chose a fill factor between 0.35 and 0.72 . But as we would like to relief as much stress as possible from the manufacturing process, whose main limitation is the aspect ratio, we decided to proceed with $f=0.5$. We also notice a dilemma between choosing a smaller wavelength (in order to reduce the necessary aspect ratio) and the difficulty to manufacture the small structures which then are just

\begin{tabular}{|c|c|c|c|c|c|c|}
\hline Material $/ \lambda$ & $f_{\max }$ & $p[\mathrm{~nm}]$ & $h\left(f_{0.5}\right)[\mathrm{nm}]$ & $h\left(f_{\max }\right)[\mathrm{nm}]$ & $A R\left(f_{0.5}\right)$ & $A R\left(f_{\max }\right)$ \\
\hline $\mathrm{SiO}_{2} / 700 \mathrm{~nm}$ & 0.55 & 250 & 3012 & 2986 & 24.1 & 26.5 \\
\hline $\mathrm{SiO}_{2} / 1400 \mathrm{~nm}$ & 0.55 & 500 & 8757 & 8685 & 35.0 & 38.6 \\
\hline $\mathrm{TiO}_{2} / 700 \mathrm{~nm}$ & 0.61 & 250 & 682 & 655 & 5.5 & 6.7 \\
\hline $\mathrm{TiO}_{2} / 1400 \mathrm{~nm}$ & 0.61 & 500 & 1491 & 1436 & 6.0 & 7.4 \\
\hline $\mathrm{Si} / 1400 \mathrm{~nm}$ & 0.67 & 500 & 580 & 538 & 2.3 & 3.2 \\
\hline
\end{tabular}

Table 1 EMA-calculations for three materials 
of about $100 \mathrm{~nm}$ in lateral extent. With the appropriate (and expensive) manufacturing technologies for very small structures and a slightly smaller wavelength than $700 \mathrm{~nm}, \mathrm{TiO}_{2}$ might also serve our purposes. But we need to keep in mind that with the EMA-approach we overestimate the refractive index for TM-polarization, meaning that during the following RCWA-simulations the etching depth will most likely become greater and with it the aspect ratio as well. For realizing and demonstrating the performance of our grating, we thus choose an infrared wavelength and the high-refractive index of silicon due to the lowest $A R$ combined with a period of $500 \mathrm{~nm}$.

\section{Phase differences}

We showed how to design a zero-order form birefringent grating with a phase difference of $\psi=\pi$ with EMA. In order to use it as a diffraction grating as shown in Fig. 3, one also needs to take into account the phase differences $\phi_{T E}$ and $\phi_{T E}$. It is not sufficient to ensure that $\psi=\pi$, but at the same time $\phi_{T E}=2 \pi$ (and $\phi_{T M}=\pi$, respectively) has to be fulfilled in order to make sure that one polarization direction (e.g. TE) does not experience diffraction. But once the material and the fill factor are determined, the form birefringence $\Delta n$ is fixed. The only remaining variable is the structure height $h$. So, as apparent in Eq. 3, we need to meet two conditions with just one parameter. That is not automatically the case, as can be seen in Fig. 5 on the left.

However, there is a solution to this problem, which can be seen in Fig. 5 on the right: We add another parameter, which practically means introducing an extra phase step. This way after choosing the parameters for $\psi$, one can easily calculate the height $h_{2}$ for yielding the proper phase steps $\phi_{T E / T M}$, following Eq. 4.

$$
\phi_{T E / T M}=\frac{2 \pi}{\lambda} \cdot\left(h_{1}\left(n_{T E / T M}-1\right)+h_{2}\left(n_{g}-1\right)\right)
$$

EMA calculations show, that for our parameter set the additional height would be $h_{2}=-30 \mathrm{~nm}$. This is almost negligible and the RCWA simulations in the following section show, that it does not seem worth taking the effort of increasing the manufacturing complexity significantly (non-binary grating) for just a marginal improvement in operation. However, for other parameter sets, adding this step can be crucial for the functionality.

\section{Calculating height and efficiencies}

Most of the parameters have already been fixed in the discussion above: $\lambda=1400 \mathrm{~nm}, p_{1}=500 \mathrm{~nm}$, the fill factor $f=0.5$, and the material silicon. We also calculated a height $h=580 \mathrm{~nm}$ with EMA. For our further simulations we now set $p_{2}=10 \mu \mathrm{m}$ and a fill factor of 0.5 for the diffraction grating. This way we yield a symmetric diffraction pattern with a diffraction angle of $8^{\circ}$ and a sufficient number of ridges (10) within a period, in case that during fabrication the ridges on both edges are not cast properly. Of course, other parameters can be chosen, depending on the application.

As we discussed the inaccuracies of our EMA-approach for our relatively large structures compared to the period and for calculating the expected diffraction efficiencies, we now carry on with rigorous simulations. To this end we used the grating toolbox in the software VirtualLab and the software MC Grating. One can execute parameter runs with $h$ as a free variable to choose the appropriate, more accurate depth and look at the diffraction efficiencies for TE and TM. Due to the aforementioned problem of not adding an additional step height $h_{2}$, one cannot find a depth which fulfils all requirements, so we decided to choose the configuration for which the $0^{\text {th }}$ order is completely eliminated. This is the case for $h=700 \mathrm{~nm}$. We also found another promising possibility for an etching depth of $h=310 \mathrm{~nm}$. In this case, $\phi_{T E}=\pi$ and $\phi_{T M}$ is of course not zero, but becomes small. As you can see in Table 2, the expected performance is not as good as for $700 \mathrm{~nm}$, but would relieve some strain from the manufacturing process. Note, that due to the high-refractive material in air, we can expect high Fresnel losses. Shown

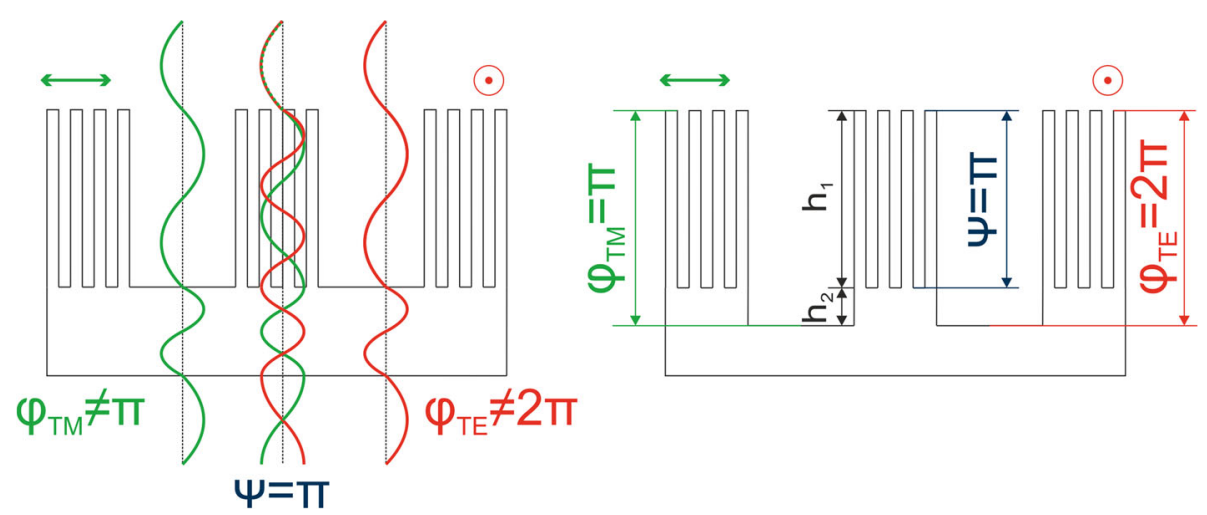

Fig. 5 Design variation: Additional step 
Table 2 RCWA-simulated diffraction efficiencies

\begin{tabular}{lllllll}
\hline Etching depth $\boldsymbol{h}$ & $\eta_{T E, 0}$ & $\eta_{T E, 1}$ & $\eta_{T E, 3}$ & $\eta_{T M, 0}$ & $\eta_{T M, 1}$ & $\eta_{T M, 3}$ \\
\hline $700 \mathrm{~nm}$ & $57.7 \%$ & $0.3 \%$ & $0.1 \%$ & $0.1 \%$ & $27.6 \%$ & $3.3 \%$ \\
$310 \mathrm{~nm}$ & $0.8 \%$ & $25.5 \%$ & $3.1 \%$ & $38.5 \%$ & $10.1 \%$ & $1.2 \%$ \\
\hline
\end{tabular}

are the simulated diffraction efficiencies in the $0^{\text {th }}, 1^{\text {st }}$, and $3^{\text {rd }}$ order. The negative orders show the same intensity and the even orders have an intensity of 0 , for it is a binary grating with $\mathrm{f}=0.5$.

The intensity in the higher orders is negligible. Those simulation results show a very strong difference in diffraction efficiency, even though it could be improved further by reducing the Fresnel losses. We decided to proceed with a height of $h=700 \mathrm{~nm}$, for which we can expect a better functionality and the aspect ratio of $A R=2.8$ seems manageable.

\section{Tolerancing}

Further simulations with respect to the wavelength give us some hints on how tolerant the system is towards manufacturing errors (etching depth). This can be verified experimentally later. Within a range of $\lambda=(1400 \pm 60) \mathrm{nm}$ we still see a high difference in diffraction efficiencies and depending on the sensibility of the system, also higher deviations may be tolerable. Also we remember our requirement of functionality over a large range of incidence angles. We carried out simulations for incidence angles of $0 \pm 45^{\circ}$. The following Fig. 6 shows the simulation results.

With this parameter set we fulfilled the requirements 1,2 and 4 . The manufacturing technique needs to ensure the reproducibility of our grating (requirement 3 ).

\section{Manufacturing}

Reliable manufacturing of subwavelength-structures is always a challenge, especially for large areas in the range of $\mathrm{cm} \times \mathrm{cm}$. We need our structures on large areas, because we eventually want to measure surfaces with high frequencies, which leads to higher reflection angles. For demonstration purposes our structure needs to be at least the size of our collimated laser beam. For our small structures it is necessary to use a technology like electron beam lithography, which is very common in research facilities, but is expensive for large areas. On the upside, we can at least cast several gratings from one e-beam-structured master if we use Soft-UV-Nanoimprint-Lithography (NIL). This technique enables us to cast structures with sizes down to just a few tens of nanometers [28]. But we are not just limited by the structure size itself, we are also limited by our aspect ratio. Therefore various steps are needed to manufacture our grating, which are described in a simplified way in the following section. All steps but the first electron-beam lithography step were carried out in-house in the cleanroom facilities at the Centre for Micro- and Nanotechnologies.

We created mask data, which contains on one wafer four different grating designs to account for possible manufacturing errors. The only difference are the fill factors of $f_{i}=0.5 ; 0.53 ; 0.56 ; 0.66$. All four gratings have a size of $2 \times 2 \mathrm{~cm}^{2}$.

Figure 7 gives a simplified overview over the various manufacturing steps, which are described in greater detail in the following paragraph.

We started with an electron-beam-structured chromium mask on fused silica, which was fabricated by Photronics MZD GmbH in Dresden. The first dry-etching pattern transfer of the chromium layer to the fused silica wafer (ICP-RIE plasma etcher SI 500; $\mathrm{CHF}_{3} / \mathrm{SF}_{6}$ ) results in our master for the following NIL-steps. This way we
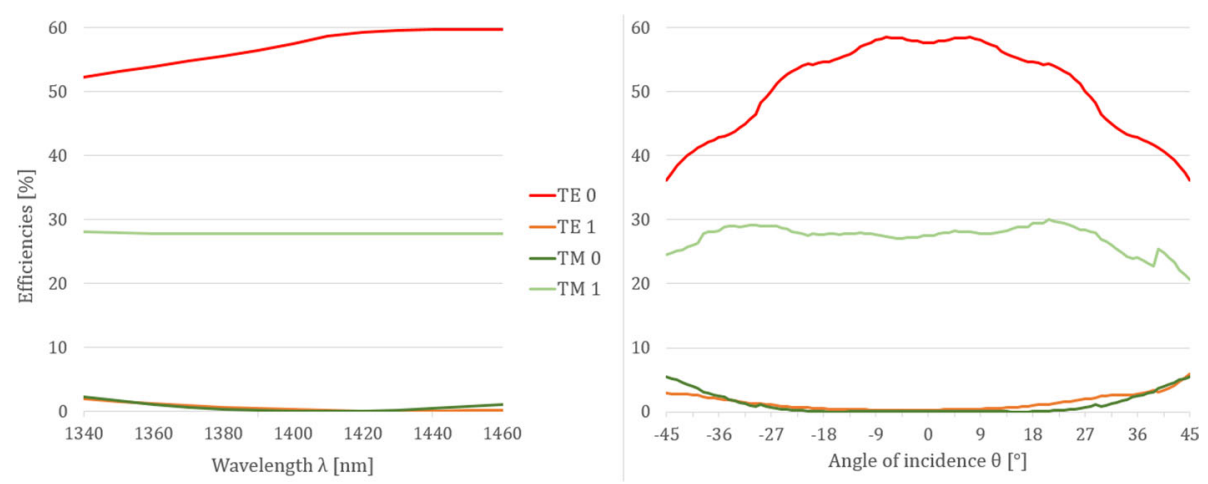

Fig. 6 Grating tolerances 


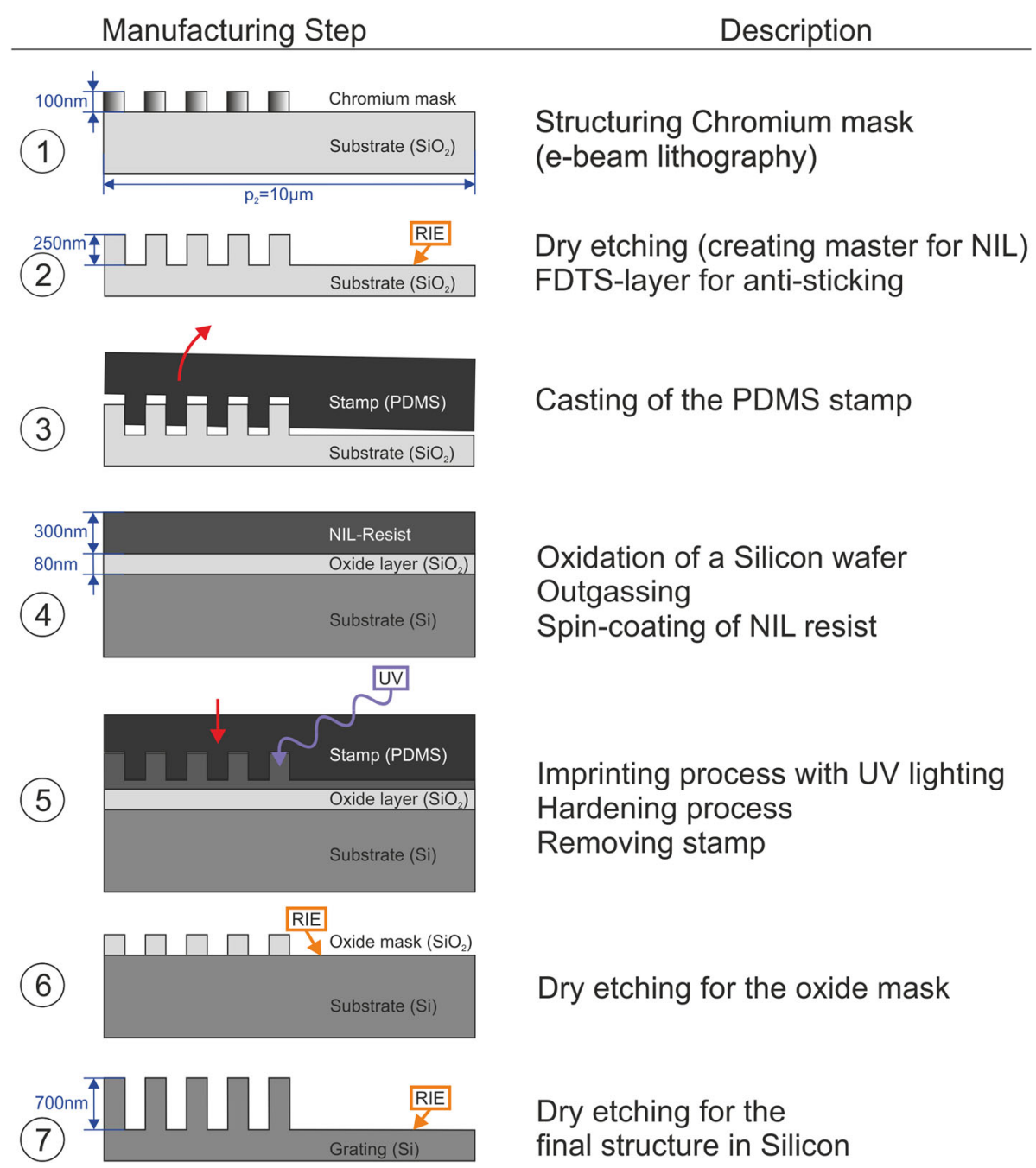

Fig. 7 Manufacturing steps

yield a relatively low-priced master for the NIL in $\mathrm{SiO}_{2}$, manufactured with a standard process, as an alternative to a more costly NIL-master in Si. It has structure depths of $250 \mathrm{~nm}$, which was confirmed with AFM-measurements.

Now the NIL-steps can follow, which can be repeated several times in order to cast numerous gratings with one master. We use vapor deposition of Perfluorodecyltrichlorosilane (FDTS) to yield an anti-sticking property of the surface of the master. The PDMS is poured onto the master. Due to the small scale of our trenches, it showed to be advantageous to dilute the PDMS with tert-butanol. After the baking process we yield the PDMS stamp. The stamp is stripped carefully from the FDTS-coated master in direction of the trenches. Forming the PDMS stamp has shown to be the most delicate step and a lot of effort went into optimizing the dilution, the outgassing in the vacuum oven and the stripping of the stamp.
We chose to use a $\mathrm{SiO}_{2}$ mask for the high selectivity of such a mask on a silicon substrate, using a fluorocarbonbased cryo etching approach. We execute a dry thermal oxidation process with a silicon wafer up to an oxide layer thickness of $80 \mathrm{~nm}$. The wafer was left in the oven for $24 \mathrm{~h}$ at temperatures of $250^{\circ} \mathrm{C}$ and we deposit a layer of Hexamethyldisilazane (HMDS) with a hotplate, which improved the adhesion of the resist significantly. On top of that we spincoat the NIL resist mr-NIL 210 (micro resist technology $\mathrm{GmbH}$ ) with a thickness of $300 \mathrm{~nm}$. Subsequently follows the imprinting process.

In the nanoimprinting tool GD-N-03 the oxidized wafer with the NIL resist layer is pressed against the stamp. The pattern from the stamp is transferred under UV lighting to the resist layer and after cooling down, the wafer can be separated from the stamp. This is also a delicate process, tuning both pressure and UV dosage. In theory, the stamp 


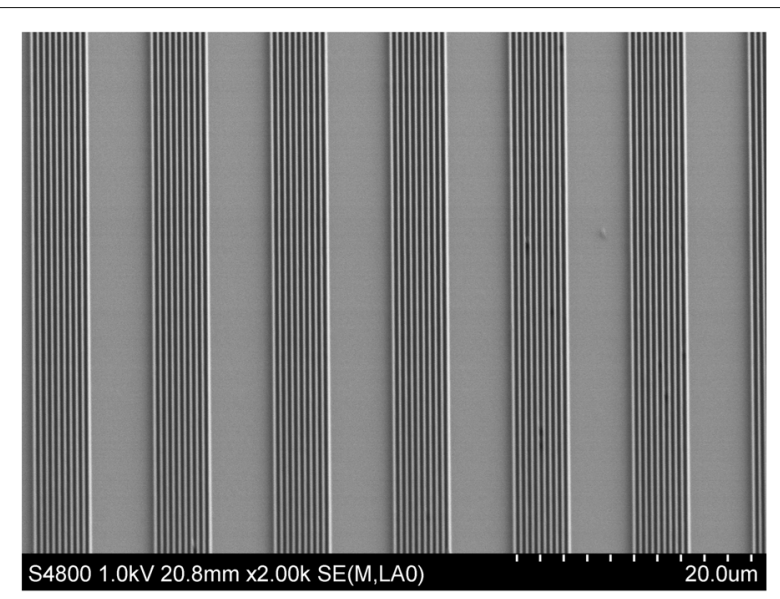

Fig. 8 SEM image of final structure in silicon

can also be reused. Up to now, in our case, when we used it for the third time, that did not lead to good results. We could see parts of the resist sticking to the PDMS stamp.

Two more etching steps are needed to yield the final structure in silicon, which can be seen in a SEM picture in Fig. 8.

The transfer from the resist pattern to the oxide layer is carried out with an ICP-RIE process, but because there is now a resist mask and not a chromium mask as in out first step, it is carried out with other process gases and parameters $\left(\mathrm{CHF}_{3} / \mathrm{Ar}\right.$ plasma etcher SI 500). The transfer from the oxide mask to the silicon wafer is achieved with cryogenic ICP-RIE $\left(\mathrm{SF}_{6} / \mathrm{O}_{2}\right.$ PlasmaPro 100 Cobra).

Even though in other works structures even smaller than ours were cast with NIL, due to our design (deep trenches over different magnitudes $[\mathrm{cm} / \mu \mathrm{m} / \mathrm{nm}]$ ) we seem to have reached a limit, as we could not satisfactorily cast the design with $f=0.66$ (i.e. trenches with a width of $165 \mathrm{~nm}$ and $A R=4.2$ ). The three other gratings were cast properly. The best result for $f=0.5$ can be seen in Fig. 8 .

For reasons of cost-effectiveness, we used a single-sided polished silicon wafer for carrying out the process development. When we yielded the most promising results, it was necessary to polish the backside of the wafer manually after the structuring. The wafer was cut into four individual gratings with a wafer saw.

\section{Experimental verification}

Of the four different manufactured gratings all but the one with $f=0.66$ were cast properly. The highest difference in diffraction efficiency however showed the one with $f=0.53$, just slightly more than the one with $f=0.56$. This hints, that the etching depth within the subwavelength structures is slightly less than the designed $700 \mathrm{~nm}$. The grating with $f=0.5$ could not eliminate the $0^{\text {th }}$ order entirely, but still shows high differences in diffraction efficiency. The results in the following paragraphs are shown for the grating with the best performance.

\section{Diffraction efficiencies}

For the experimental verification we use a supercontinuum (SC) fiber laser (NKT SuperK extreme EXB4), set to a wavelength of $\lambda=1400 \mathrm{~nm}$ (LLTFcontrast SWIR HP8). The fiber is not polarization-maintaining, so we let the light pass through a linear polarizer (Thorlabs LPIREA050-C, extinction ratio of 2349:1 for $\lambda=1400 \mathrm{~nm}$ ). The grating is illuminated from the polished back side of the wafer. The grating is fixed, the polarizer is rotated to obtain TE- and TM-polarization, respectively. The intensities of the different orders are measured with the Thorlabs power meter PM320E and the sensor S122C. The setup can be seen in Fig. 9.

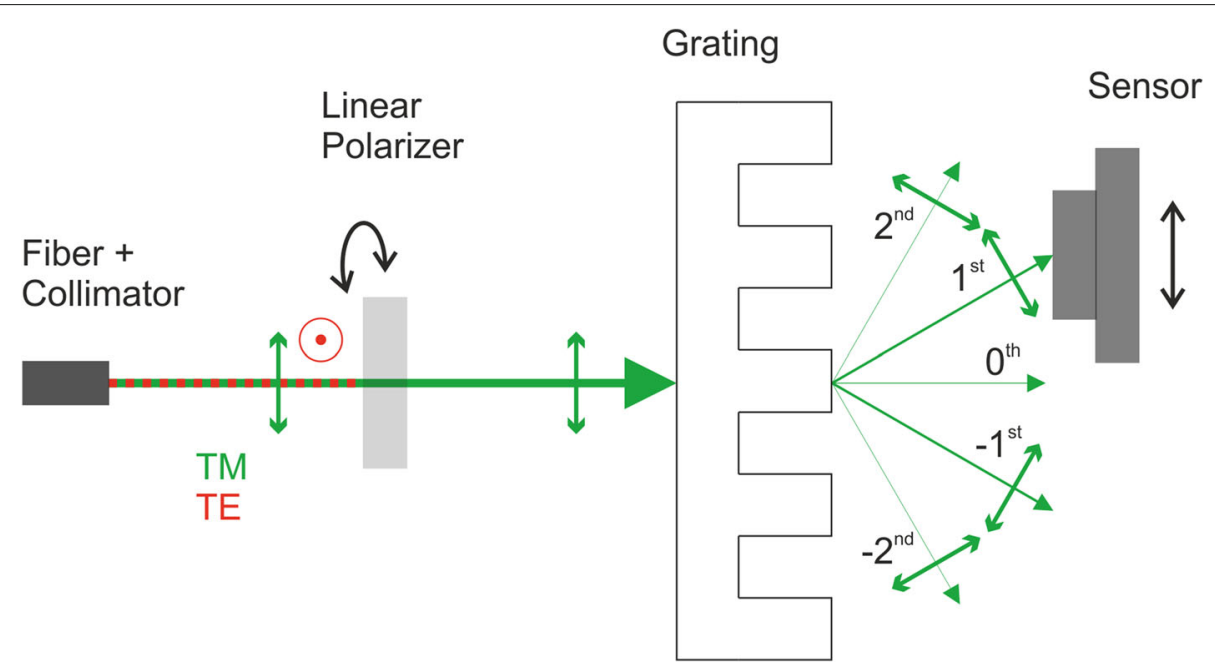

Fig. 9 Setup for measuring the efficiency 


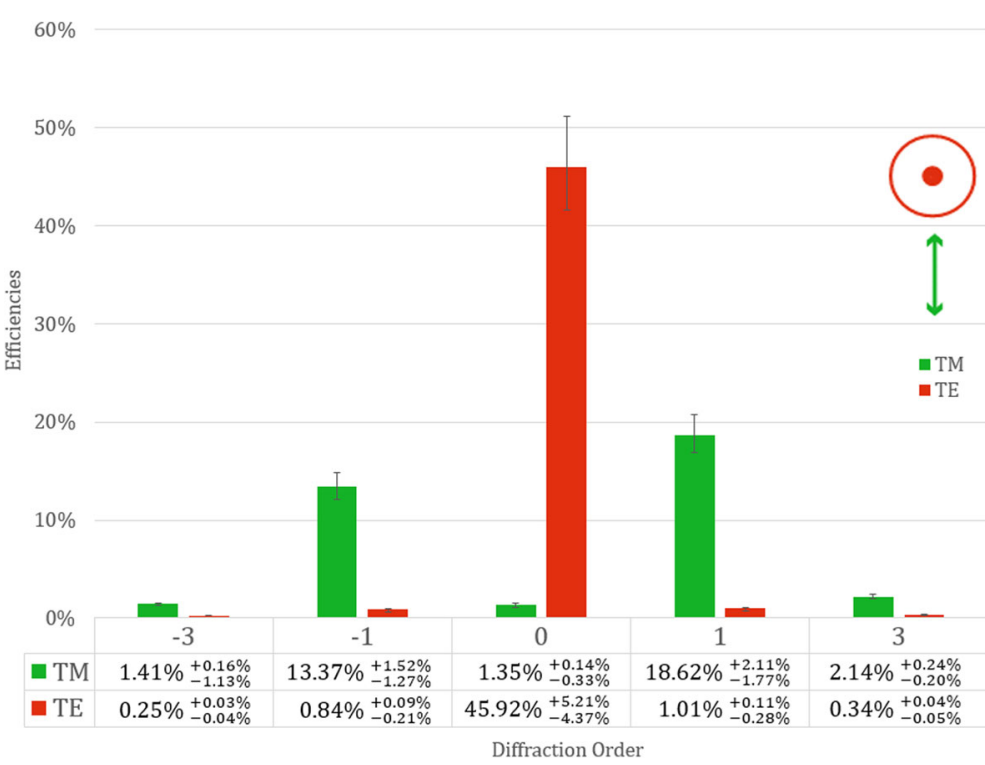

Fig. 10 Measured diffraction efficiencies

Note, that the efficiency is defined as intensity in the respective order over the intensity of the light behind the polarizer, before interacting with the grating. The Fresnel losses are very high (approximated with two even siliconair interfaces $\approx 52 \%$ ), so the results could be increased significantly by adding an anti-reflective layer. But still we can clearly see, experimentally verified, a strong polarizing beamsplitter (Fig. 10).

The measurement accuracy shown in Fig. 10 was estimated based on the following: The sensor has a measurement uncertainty of $\pm 5 \%$. Moreover, the accurate placement of the elements to each other play a key role. It was estimated, that it is possible to align the polarization plane within a range of $\pm 5^{\circ}$ relative to the grating. This misalignment shows the far greater effect on the measurement uncertainty than the extinction ratio of the polarizer, which in our case causes a negligible error. Furthermore, the deviation of the incidence angle $\theta$ was estimated at $\pm 1^{\circ}$. The influence of non-vertical incidence is shown in Fig. 6 (simulation) and Fig. 11 (measurement) separately. Besides its beamsplitting characteristics, the device shows some asymmetry in efficiency between the positive and negative orders. The following three effects might cause this effect: Firstly, the incidence angle might not be exactly perpendicular to the grating. Simulations show, that this causes some deviation in symmetry for the subwavelength structures. Secondly, the backside of the wafer might not be perfectly parallel to the etched surface on the other side, which might have been caused by the polishing step. This leads to a phase wedge and this clearly
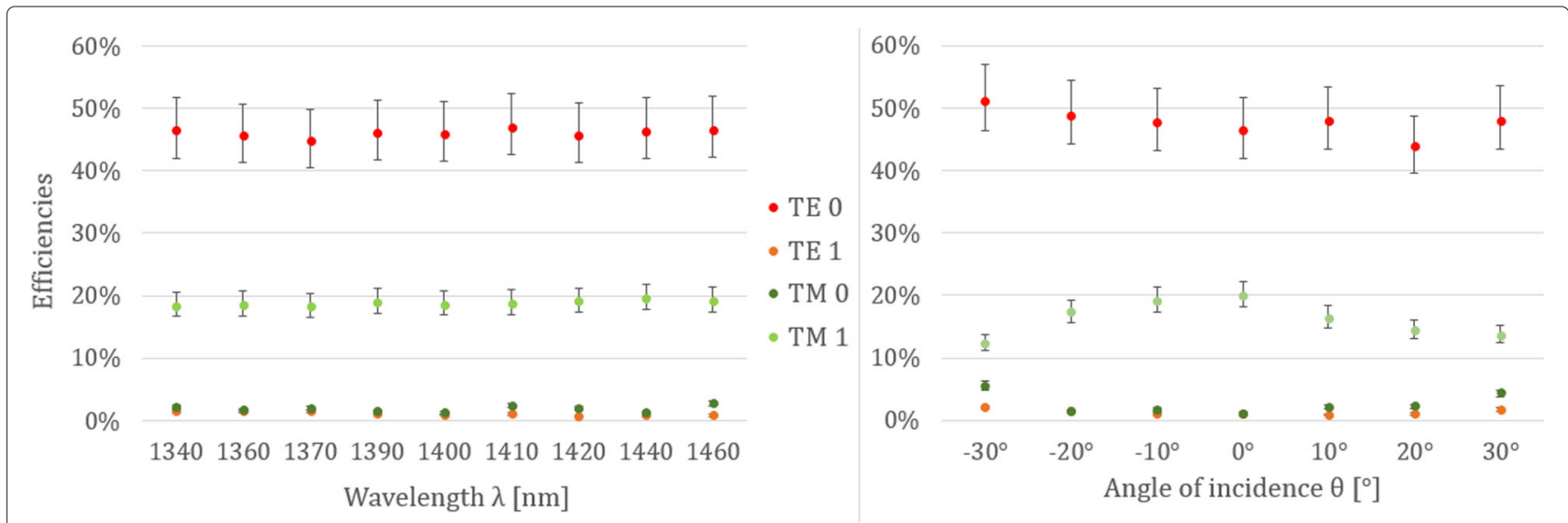

Fig. 11 Measured tolerances 


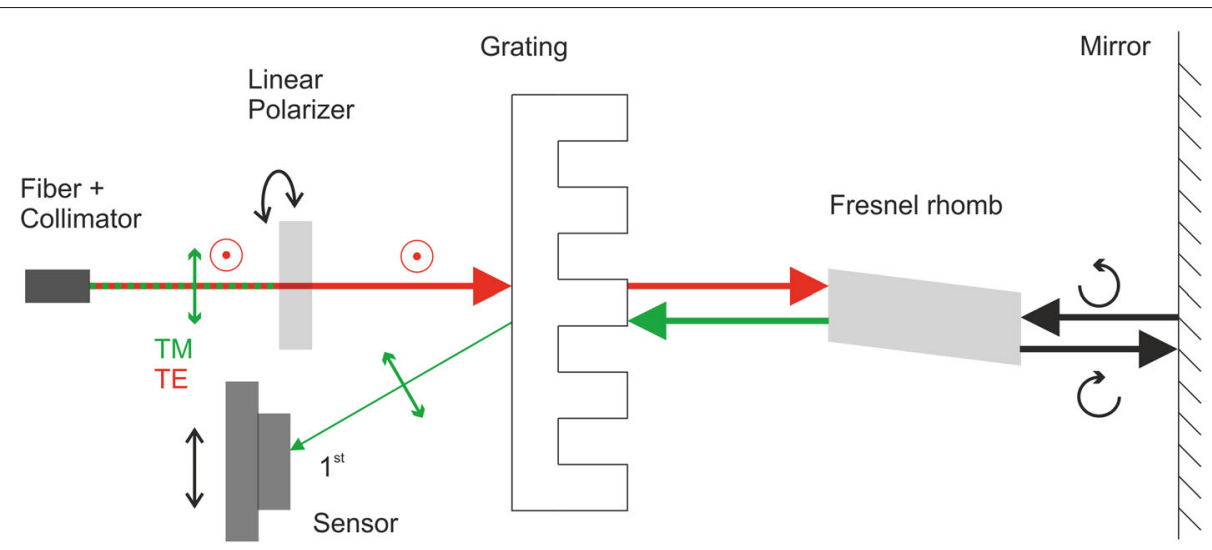

Fig. 12 Complete measurement setup

can cause the effect in Fig. 10. Thirdly, we now, generally speaking, that the etch rate on larger areas is higher than the rate on smaller areas. So it is likely that the etching depth between the subwavelength structures (with a width of $250 \mathrm{~nm}$ ) is slightly smaller than the etching depth on the larger, $5 \mu \mathrm{m}$ wide areas. The effect of the etching depth variation has been simulated with RCWA algorithms. The simulations show, that a deviation in etching depth of just a few nanometers results in a minor asymmetry in diffraction efficiency. Current investigations are focussing on a more detailed understanding of this effect. For optimized grating performance it will be necessary in future experiments to take into account the different etching rates.

\section{Tolerances}

Within a range of $\lambda=(1400 \pm 60) \mathrm{nm}$ we do not see a large difference in diffraction efficiencies, meaning it can be used with more than just one wavelength and hints, that we have some stability against deviations in the etching depth (see Fig. 11).

Moreover, to verify the functionality over a large range of incidence angles, measurements were carried out for an incidence angle $\theta=-30^{\circ} \ldots+30^{\circ}$, which was the maximum angle our experimental setup allowed. Note, that it is important to illuminate from the back side of the polished wafer for high angles, as otherwise one might run into problems with total internal reflection for high-refractive material. This can also be done within a complete system as shown in Figs. 1 and 12, as the illumination is perpendicular to the grating.

\section{Complete setup}

We now want to compare the efficiencies of an amplitude grating in a setup comparable to Fig. 1 to the efficiencies of our grating. To this end we use a similar setup, as shown in Fig. 2, and set up an experiment where the light passes the grating twice. In Fig. 12 one can see the complete setup. As in our previous measurements, we polarize the light coming from our SC-Laser before it passes the grating from the polished back side. We set TE-polarization, so that ideally all of the light passes. As shown in Table 3, we already lose a lot of intensity due to Fresnel reflections. We use a Fresnel rhomb, which acts as a broadband quarterwave plate. First we measure the intensity in the $0^{\text {th }}$ order (TE) directly behind the grating and the $1^{\text {st }}$ and $-1^{\text {st }}$ order (TM) of the light, which was reflected by a silver-coated mirror and went through the grating (now with rotated state of polarization) a second time.

The results are shown in Table 3. Note, that our results include both Fresnel losses of the grating and the Fresnel rhomb and inaccuracies, which occurred during the manufacturing process; also losses at the mirror and deviations caused by adjustment inaccuracies. We compare these results to the estimations from our RCWA-results (Table 2), which just include the Fresnel losses of an otherwise ideal grating and no other potential error sources.

It may be possible to further optimize the manufacturing process, which might lead to further efficiency increases. But the far greater gain in efficiency could be achieved by adding anti-reflective coatings. Even if for the structured side of the wafer this might be challenging, only executing that on the flat side of the wafer would be a huge improvement. Nevertheless it can be seen in comparison

Table 3 Comparison between grating types

\begin{tabular}{llll}
\hline Grating type & $\eta_{\mathbf{0}}$ & $\boldsymbol{\eta}_{\mathbf{1}}$ & $\boldsymbol{\eta}_{\mathbf{- 1}}$ \\
\hline $\begin{array}{l}\text { Amplitude grating }(\mathrm{f}=0.5) \\
\text { (theoretically) }\end{array}$ & $25 \%$ & $2.5 \%$ & $2.5 \%$ \\
$\begin{array}{l}\text { Hybrid subwavelength grating } \\
\text { (theoretically) }\end{array}$ & $57.7 \%$ & $15.9 \%$ & $15.9 \%$ \\
$\begin{array}{l}\text { Hybrid subwavelength grating } \\
\text { (experimentally) }\end{array}$ & $49.0_{-4.7}^{+5.2 \%}$ & $6.4_{-0.4}^{+0.9 \%}$ & $4.9_{-0.3}^{+0.7 \%}$ \\
\hline
\end{tabular}


with the theoretically maximal achievable efficiencies of an amplitude grating, that our grating shows an improved performance.

\section{Conclusion}

We presented an approach for designing subwavelengthstructured, binary, polarizing diffraction gratings, which act as a polarizing beamsplitter over a long range of incidence angles. The design strategy is closely related to previously published concepts $[24,25]$. We simulated and realized a grating in silicon. For manufacturing we chose the Nanoimprint-Lithography, for it can be used to reproduce subwavelength structures in an economical way on large areas. We adapted and developed processes for the whole production chain, using NIL and dry etching processes. We verified our simulated results experimentally. We presented an application for such a grating for the measurement of freeform surfaces and showed, that our grating improves the efficiency of this optical system. Also, there are several options to further improve the efficiency of our grating, mainly by adding anti-reflective coating but also through further optimizations in the production process. Our design approach and NIL technique can be used to realize other polarization-dependent diffractive optics, and opens the door for further development of specialized polarization-optical, compact components.

\section{Abbreviations \\ AFM: Atomic force microscope; EMA: Effective medium approximation; ICP: Inductively coupled plasma; FDTS: Perfluorodecyltrichlorosilane; HMDS: Hexamethyldisilazane; NIL: Nanoimprint-Lithography; PDMS: Polydimethylsiloxane; RCWA: Rigorous coupled-wave analysis; RIE: Reactive ion etching; SC: Super continuum; SEM: Scanning electron microscope; TE: Transversal electric; TM: Transversal magnetic; UV: Ultraviolet}

\section{Acknowledgements}

Many thanks to Lars Dittrich, Shuhao Si and Shraddha Supreeti for sharing their experience with NIL manufacturing methods and to Nikolay Lukin, who carried out some measurements with the complete setup.

\section{Authors' information}

The work by Yannick Bourgin was carried out while he was working at the TU Ilmenau.

\section{Authors' contributions}

$J W, Y B$ and StS worked mainly on the concept, the design and the simulations. The first author also carried out the experimental verification and interpretation of the data. PF and AB were responsible for the process development and manufacturing. All authors read and approved the final manuscript.

\section{Funding}

Deutsche Forschungsgemeinschaft (DFG), Graduiertenkolleg 2182 "NanoFab" Deutsche Forschungsgemeinschaft (DFG), Project No. 390737909 "Wavesynth" Open Access funding enabled and organized by Projekt DEAL.

\section{Availability of data and materials}

Upon request to the corresponding author.

\section{Declarations}

\section{Competing interests}

The authors declare that they have no competing interests.

\section{Author details}

'Department Technical Optics, Institute for Micro- and Nanotechnologies, Technische Universität IImenau, 98693 IImenau, Germany. ${ }^{2}$ siOPTICA GmbH, Moritz-von-Rohr-Straße 1a, 07745 Jena, Germany.

Received: 8 December 2020 Accepted: 28 February 2021

Published online: 24 March 2021

\section{References}

1. Bichra, M., Sabitov, N., Meinecke, T., Sinzinger, S.: Wavefront sensing by numerical evaluation of diffracted wavefields. Appl. Opt. 56, 13-22 (2017)

2. Bichra, M., Meinecke, T., Fesser, P., Müller, L., Hoffmann, M., Sinzinger, S.: Freeform characterization based on nanostructured diffraction gratings. Appl. Opt. 57, 3808-3816 (2018)

3. Wüster, J., Bourgin, Y., Feßer, P., Sinzinger, S.: Nano-structured diffraction gratings as polarizing beam splitters under vertical incidence. In: Saastamoinen, K. (ed.) Abstracts of the EOS Topical Meeting on Diffractive Optics, pp. 20-21. European Optical Society (EOS), Jena, (2019)

4. Wüster, J., Bourgin, Y., Fe $\beta$ er, P., Si, S., Sinzinger, S.: Nanostrukturierte beugungsgitter als angepasste integrierbare polarisationsstrahlteiler. In: Proceedings of the MikroSystemTechnik Kongress. VDE, pp. 468-471. VDE Verlag, Berlin, (2019)

5. Bartelt, H., Glaser, T., Schröter, S.: Modelling and characterization of optical high frequency gratings. Optik. 112, 283-288 (2001)

6. Clausnitzer, T., Kämpfe, T., Kley, E.-B., Tünnermann, A., Tishchenko, A., Parriaux, O.: Investigation of the polarization-dependent diffraction of deep dielectric rectangular transmission gratings illuminated in Littrow mounting. Appl. Opt. 46, 819-826 (2007)

7. Zheng, J., Zhou, C., Wang, B., Feng, J.: Beam splitting of low-contrast binary gratings under second Bragg angle incidence. J. Opt. Soc. Am. A. 25, 1075-1083 (2008)

8. Enger, R. C., Case, S. K.: Optical elements with ultrahigh spatial-frequency surface corrugations. Appl. Opt. 22, 3220-3228 (1983)

9. Stork, W., Streibl, N., Haidner, H., Kipfer, P.: Artificial distributed-index media fabricated by zero-order gratings. Opt. Lett. 16, 1921-1923 (1991)

10. Haidner, H., Kipfer, P., Storck, W., Streibl, N.: Zero-order gratings used as an artificial distributed index medium. Optik. 89, 107-112 (1992)

11. Lalanne, P., Lemercier-lalanne, D.: On the effective medium theory of subwavelength periodic structures. J. Mod. Opt. 43, 2063-2085 (1996)

12. Lalanne, P., Astilean, S., Chavel, P., Cambril, E., Launois, H.: Blazed binary subwavelength gratings with efficiencies larger than those of conventional échelette gratings. Opt. Lett. 23, 1081-1083 (1998)

13. Lalanne, P., Astilean, S., Chavel, P., Cambril, E., Launois, H.: Design and fabrication of blazed binary diffractive elements with sampling periods smaller than the structural cutoff. J. Opt. Soc. Am. A. 16, 1143-1156 (1999)

14. Cescato, L. H., Gluch, E., Streibl, N.: Holographic quarterwave plates. Appl. Opt. 29, 3286-3290 (1990)

15. Kikuta, H., Ohira, Y., Iwata, K.: Achromatic quarter-wave plates using the dispersion of form birefringence. Appl. Opt. 36, 1566-1572 (1997)

16. Nordin, G., Deguzman, P.: Broadband form birefringent quarter-wave plate for the mid-infrared wavelength region. Opt. Express. 5, 163-168 (1999)

17. Stock, C., Siefke, T., Zeitner, U. D., Kley, E.-B.: Nano-optical quarter-wave plates for applications in the visible wavelength regime: fabrication, tolerances and in-situ process control. In: Engineering for a Changing World: Proceedings; 59th IImenau Scientific Colloquium, Technische Universität Ilmenau, (2017)

18. Päivänranta, B., Passilly, N., Pietarinen, J., Laakkonen, P., Kuittinen, M., Tervo, J.: Low-cost fabrication of form-birefringent quarter-wave plates. Opt. Express. 16, 16334-16342 (2008)

19. Isano, T., Kaneda, Y., Iwakami, N., Ishizuka, K., Suzuki, N.: Fabrication of half-wave plates with subwavelength structures. Jpn. J. Appl. Phys. 43, 5294-5296 (2004)

20. Lopez, A. G., Craighead, H. G.: Wave-plate polarizing beam splitter based on a form-birefringent multilayer grating. Opt. Lett. 23, 1627-1629 (1998)

21. Tyan, R.-C., Salvekar, A. A., Chou, H.-P., Cheng, C.-C., Scherer, A., Sun, P.-C., $\mathrm{Xu}$, F., Fainman, Y.: Design, fabrication, and characterization of form-birefringent multilayer polarizing beam splitter. J. Opt. Soc. Am. A. 14, 1627-1636 (1997)

22. Lalanne, P., Hazart, J., Chavel, P., Cambril, E., Launois, H.: A transmission polarizing beam splitter grating. Opt. Lett. 1, 215-219 (1999) 
23. Hasman, E., Bomzon, Z., Niv, A., Biener, G., Kleiner, V.: Polarization beam-splitters and optical switches based on space-variant computer-generated subwavelength quasi-periodic structures. Opt. Commun. 209, 45-54 (2002)

24. Haidner, H., Dias, D., Wang, L. L., Tschudi, T.: Binary subwavelength structures/resonance gratings as polarization elements. Pure Appl. Opt. J. Eur. Opt. Soc. A. 7, 1347-1361 (1998)

25. Xu, F., Tyan, R. C., Sun, P. C., Fainman, Y., Cheng, C. C., Scherer, A.: Form-birefringent computer-generated holograms. Opt. Lett. 21, 1513-1515 (1996)

26. Bell, J. M., Derrick, G. H., McPhedran, R. C.: Diffraction gratings in the quasi-static limit. Opti. Acta Int. J. Opt. 29, 1475-1489 (1982)

27. Haidner, H.: Theorie der subwellenlängenstrukturen für diffraktive optische elemente. PhD thesis. Friedrich-Alexander-Universität Erlangen-Nürnberg (1994). http://d-nb.info/941006697

28. Si, S., Dittrich, L., Hoffmann, M.: The NanoTuFe-Fabrication of large area periodic nanopatterns with tunable feature sizes at low cost. Microelectron. Eng. 180, 71-80 (2017)

\section{Publisher's Note}

Springer Nature remains neutral with regard to jurisdictional claims in published maps and institutional affiliations.

\section{Submit your manuscript to a SpringerOpen ${ }^{\odot}$ journal and benefit from:}

- Convenient online submission

- Rigorous peer review

- Open access: articles freely available online

- High visibility within the field

- Retaining the copyright to your article

Submit your next manuscript at $\gg$ springeropen.com 